\title{
O'NEIL'S THRESHOLD FOR NONLINEAR LANDAU DAMPING AND PHASE TRANSITION IN THE WAVE-PARTICLE SYSTEM
}

\author{
MARIE-Christine Firpo ${ }^{1}$ And Yves Elskens ${ }^{1}$
}

\begin{abstract}
Many important nonlinear phenomena of plasma physics emerge from the study of systems involving only a finite number of waves. One famous example, first treated by O'Neil, is the breakdown of the linear analysis of collisionless Landau damping due to trapping, in the case of a single long wavelength Langmuir wave. Using a statistical mechanics treatment applied to a wave-particle selfconsistent Hamiltonian model, we show that the dynamical threshold predicted by O'Neil can be related to the critical point of a second order phase transition, with the amplitude of the wave behaving as an order parameter. This analysis is self-consistent and, dynamically, a time-algebraic decay of the wave may signal the vicinity of the critical point.

Résumé. Beaucoup de phénomènes importants de physique des plasmas ressortent de l'étude de systèmes ne comportant qu'un nombre fini d'ondes. Un exemple fameux, traité par O'Neil, est la mise en défaut de l'analyse linéaire de l'amortissement non-collisionnel de Landau à cause du piégeage, dans le cas d'une seule onde de Langmuir de grande longueur d'onde. Par un traitement de mécanique statistique d'équilibre appliqué à un modèle auto-cohérent onde-particule, on montre que le seuil dynamique prédit par O'Neil peut être relié au point critique d'une transition de phase du deuxième ordre admettant l'amplitude de l'onde comme paramètre d'ordre. Cette analyse est auto-cohérente, et, dynamiquement, l'approche du point critique pourrait être signalée par une décroissance algébrique de l'amplitude de l'onde.
\end{abstract}

AMS Subject Classification. 82B05, 82B26, 82C40.

\section{INTRODUCTION}

Landau's rigorous treatment of the initial value problem [1] associated to the Vlasov-Poisson set of equations

$$
\begin{gathered}
\frac{\partial f}{\partial t}(x, v, t)+v \frac{\partial f}{\partial x}(x, v, t)+\frac{q}{m} E(x, t) \frac{\partial f}{\partial v}(x, v, t)=0 \\
\frac{\partial E}{\partial x}=\frac{q}{\epsilon_{0}}\left(\int f d v\right)-\frac{q_{i} N_{0}}{\epsilon_{0}}
\end{gathered}
$$

Keywords and phrases: Landau damping, Kinetic equations, Phase transitions, Collisionless plasmas

${ }^{1}$ Equipe turbulence plasma de l'UMR 6633 CNRS-Université de Provence, case 321, Centre de Saint-Jérôme, av. escadrille Normandie-Niemen, F-13397 Marseille cedex 20

e-mail: firpo@newsup.univ-mrs.fr,elskens@newsup.univ-mrs.fr

(c) EDP Sciences, SMAI 2001 
linearized around any Vlasov equilibrium $F_{0}(v)$, with $f(x, v, t)=F_{0}(v)+f_{1}(x, v, t)$, first evidenced the possibility of collisionless damping in plasmas. It is interesting to note that, through the correct treatment of the poles occuring in the dispersion function, this analysis stresses the importance of particles whose velocities are close to the phase velocities of normal modes. Landau's seminal analysis is however strictly linear and its validity is generically doomed to fail as time increases because the linear hypothesis $\left|\partial f_{1} / \partial v\right| \ll\left|d F_{0} / d v\right|$ breaks due to particle trapping. Therefore the full, intrinsically non-linear, initial value problem for Vlasov-Poisson equations remains an open issue. Lately, the related fate of the time-asymptotic behavior of the electric field has been much debated in several places $[2,3,4,5]$.

Parallel to the classical kinetic Vlasov-Poisson treatment, another approach to the damping of Langmuir waves originates in Dawson's [6] and O'Neil's [7] works, wishing to emphasize the physical mechanism at work, namely wave-particle interaction as well as a possible trapping description. This approach relies on dividing the plasma electrons in two groups : bulk particles that simply support plasma waves and quasi-resonant ("tail") particles, whose velocities are close to wave velocities, that effectively interact with the waves.

Let us consider the simplest case of $N$ identical (tail) particles moving on the interval of length $L=2 \pi$ with periodic boundary conditions $\mathbb{T}:=\mathbb{R} /(2 \pi \mathbb{Z})$, with unit mass and charge, and respectively position $x_{r}$ and momentum $p_{r}$, interacting with a single wave of natural frequency $\omega_{0}$, unit wave number, phase $\theta$ and intensity I. Then wave-particle interaction can be modelized by the self-consistent Hamiltonian $[8,9,10]$

$$
H=\sum_{r=1}^{N} \frac{p_{r}^{2}}{2}+\omega_{0} I-\sqrt{\frac{\eta \omega_{0}^{3}}{N}} \sum_{r=1}^{N} \sqrt{2 I} \cos \left(x_{r}-\theta\right) .
$$

This reduction was put in a rigorous form in Ref. [11] and we shall analyse model (3) further below.

Many important nonlinear phenomena of plasma physics emerge from the study of systems involving only a finite number of waves and one famous example, first treated by O'Neil [7], is the breakdown of the linear analysis of Landau damping due to trapping, in the case of a single long wavelength Langmuir wave. O'Neil announces a dynamical threshold conditionning the long-time evolution of the wave : if the linear damping rate $\left|\gamma_{\mathrm{L}}\right|^{-1}$ is much shorter than the nonlinear trapping time $\tau_{B 0}$, then the wave will not have enough time to feel nonlinearities and will be damped to zero. On the contrary, if $\left|\gamma_{\mathrm{L}}\right|^{-1}$ is of the order of or larger than $\tau_{B 0}$, the dynamics will be controlled by the nonlinearities (trapping). In this contribution, we shall consider this case through a statistical mechanics treatment of our Hamiltonian model and revisit, in a self-consistent way, the longstanding qualitative picture of O'Neil for nonlinear Landau damping. This approach has already been exposed in Ref. [12] where we showed that the damping regime is generically associated to a second order phase transition.

Our aim here is to relate this phase transition to O'Neil's prediction for the time-asymptotic regime. More specifically, given an initial intensity of the wave, we consider as quasi-resonant particles the particles having velocities within a relevant range from the wave phase velocity. We only consider initial linear decreasing distribution functions $f_{0}(v)$ for this particles. The linear Landau damping rate $\gamma_{\mathrm{L}}$ is then directly proportional to their slope and will be our control parameter. We show that there exists a critical damping rate $\gamma_{\mathrm{Lc}}$ such that, if $\left|\gamma_{\mathrm{L}}\right|>\left|\gamma_{\mathrm{Lc}}\right|$, the wave will asymptotically damp to zero, whereas if $\left|\gamma_{\mathrm{L}}\right|<\left|\gamma_{\mathrm{Lc}}\right|$ the time-asymptotic intensity of the wave will remain finite with particles trapped in the wave field.

\section{The MEAN-FIELD WAVE PARTICLE MODEL}

Let us first sketch the essential properties of the Hamiltonian model (3). The first term consists in the kinetic energy of the particles, the second is the energy of the free wave (harmonic oscillator) and the last one is the coupling interaction between the particles and the wave. The coupling parameter $\eta$ denotes the small ratio of the tail density over the plasma density, consistent to the approximation of weak Langmuir turbulence. The frequency of the free wave is $\omega_{0}$, which, due to the long wavelength approximation, can be identified to the plasma frequency $\omega_{\mathrm{P}}$ and provides the unit time scale $\omega_{0}^{-1}=1$ from here on. 
It is important to note the mean-field nature of model (3). Actually, the equation of motion of any particle $r$ reads

$$
\ddot{x}_{r}=-\sqrt{2 \eta I / N} \sin \left(x_{r}-\theta\right)
$$

that is the equation of a pendulum evolving in the self-consistent mean field $I$ and angle $\theta$.

The natural scaling [14] for the wave intensity $I$ in the kinetic limit $N \rightarrow \infty$ can be simply deduced from equation (4). In our mean-field model, the acceleration felt by any particle should remain finite in this limit so that $I=\mathcal{O}(N)$, defining the associated intensive kinetic quantity as $\psi:=I / N$.

The Hamiltonian (3) admits two constants of motion, the total energy $E=H$ and the total momentum $P=\sum_{r=1}^{N} p_{r}+I$. We define the intensive associated quantities $h:=H / N$ and $\sigma:=P / N$. They will be used in the following derivation of equilibrium Gibbs predictions.

\section{Equilibrium STATISTICAL MECHANICS}

As energy $E$ and momentum $P$ are conserved quantities, we shall operate in a generalized Gibbs-Boltzmann ensemble with partition function

$$
Z_{\mathrm{c}}(\beta, \lambda, N)=\int \exp (-\beta H+\lambda P) \prod_{r=1}^{N} d p_{r} d x_{r} d I d \theta,
$$

where $\beta=T^{-1}$ is the reciprocal of thermodynamical temperature $\left(k_{\mathrm{B}}=1\right)$ defined through

$$
H=\langle H\rangle_{\mathrm{c}}=-\partial_{\beta} \ln Z_{\mathrm{c}}
$$

and $\lambda$ is similarly the Lagrange multiplier associated to the total momentum with

$$
P=\langle P\rangle_{\mathrm{c}}=\partial_{\lambda} \ln Z_{\mathrm{c}}
$$

One obtains

$$
Z_{\mathrm{c}}(\beta, \lambda, N)=2 \pi\left(\frac{2 \pi}{\beta}\right)^{N / 2} \exp \left(N \frac{\lambda^{2}}{2 \beta}\right) \times \int_{0}^{\infty} \exp \left\{N\left[(\lambda-\beta) \psi+\ln I_{0}(\beta \sqrt{2 \eta \psi})\right]\right\} N d \psi
$$

where $I_{0}$ is the modified Bessel function. With the change of variable

$$
\varphi=\beta \sqrt{2 \eta \psi}
$$

one gets

$$
Z_{\mathrm{c}}(\beta, \lambda, N)=\frac{2 \pi}{\eta \beta^{2}}\left(\frac{2 \pi}{\beta}\right)^{N / 2} \exp \left(N \frac{\lambda^{2}}{2 \beta}\right) \times \int_{0}^{\infty} \exp \{-N g(\varphi, \beta, \lambda)\} N \varphi d \varphi
$$

where

$$
g(\varphi, \beta, \lambda)=(\beta-\lambda) \frac{\varphi^{2}}{2 \eta \beta^{2}}-\ln I_{0}(\varphi)
$$


In the limit of large $N$, one evaluates (8) through the saddle point method and looks for the minimum of $g$ with

$$
\frac{\partial g}{\partial \varphi}=\varphi\left[\frac{\beta-\lambda}{\eta \beta^{2}}-\frac{I_{1}(\varphi)}{\varphi I_{0}(\varphi)}\right]=0
$$

If

$$
\partial^{2} g /\left.\partial \varphi^{2}\right|_{\varphi=0}=\frac{\beta-\lambda}{\eta \beta^{2}}-\frac{1}{2}>0
$$

then the minimum is attained in $\varphi=0$, else it is attained in $\varphi^{*}(\beta, \lambda)>0$ defined implicitly by

$$
\frac{I_{1}\left(\varphi^{*}\right)}{\varphi^{*} I_{0}\left(\varphi^{*}\right)}=\frac{\beta-\lambda}{\eta \beta^{2}}
$$

For the first case $(\varphi=0)$, the partition function reads :

$$
Z_{\mathrm{c}}(\beta, \lambda, N)=\frac{2 \pi N}{\eta \beta^{2}}\left(\frac{2 \pi}{\beta}\right)^{N / 2} \exp \left(\frac{N \lambda^{2}}{2 \beta}\right)\left(\left.2 N \frac{\partial^{2} g}{\partial \varphi^{2}}\right|_{\varphi=0}\right)^{-1}
$$

and

$$
\begin{gathered}
\sigma=N^{-1}\langle P\rangle_{\mathrm{c}}=\frac{\lambda}{\beta}+\mathcal{O}\left(N^{-1}\right) . \\
h=N^{-1}\langle H\rangle_{\mathrm{c}}=\frac{\lambda^{2}}{2 \beta^{2}}+\frac{1}{2 \beta}+\mathcal{O}\left(N^{-1}\right)=\frac{T}{2}+\frac{\sigma^{2}}{2}+\mathcal{O}\left(N^{-1}\right) .
\end{gathered}
$$

For the second case $\left(\varphi^{*}(\beta, \lambda)>0\right)$, the partition function is

$$
\begin{aligned}
Z_{\mathrm{c}}(\beta, \lambda, N) & =\frac{2 \pi N}{\eta \beta^{2}}\left(\frac{2 \pi}{\beta}\right)^{N / 2} \exp \left(N \frac{\lambda^{2}}{2 \beta}-N g\left(\varphi^{*}, \beta, \lambda\right)\right) \times \int_{0}^{\infty} \exp \left\{-\left.\frac{N}{2}\left(\varphi-\varphi^{*}\right)^{2} \frac{\partial^{2} g}{\partial \varphi^{2}}\right|_{\varphi^{*}>0}\right\} \varphi d \varphi \\
& \simeq \frac{2 \pi N}{\eta \beta^{2}}\left(\frac{2 \pi}{\beta}\right)^{N / 2} \varphi^{*} \sqrt{\frac{\pi}{\left.2 N \frac{\partial^{2} g}{\partial \varphi^{2}}\right|_{\varphi^{*}>0}}} \times\left(1+\operatorname{erf}\left(\varphi^{*}\right)\right) \exp \left(N \frac{\lambda^{2}}{2 \beta}-N g\left(\varphi^{*}, \beta, \lambda\right)\right)
\end{aligned}
$$

Thus

$$
\begin{gathered}
\sigma=\frac{\lambda}{\beta}+\frac{\varphi^{* 2}}{2 \eta \beta^{2}} \Rightarrow \frac{\lambda}{\beta}=\sigma-\frac{\varphi^{* 2}}{2 \eta \beta^{2}} \\
h=\frac{1}{2 \beta}+\frac{\lambda^{2}}{2 \beta^{2}}+\frac{\partial g\left(\varphi^{*}(\beta, \lambda), \beta, \lambda\right)}{\partial \beta}=\frac{1}{2 \beta}+\frac{\lambda^{2}}{2 \beta^{2}}-\frac{\varphi^{* 2}}{2 \eta \beta^{2}}+\frac{\lambda}{\beta} \frac{\varphi^{* 2}}{\eta \beta^{2}}
\end{gathered}
$$

Let us now return to the meaning of condition (8). This is equivalent to the two conditions $1>\sigma$ and $T>T_{\mathrm{c}}$ where we define $T_{\mathrm{c}}$ as

$$
T_{\mathrm{c}}:=\frac{\eta}{2(1-\sigma)}
$$


In this regime, the Gibbs average of the wave intensity is

$$
\left.\langle I\rangle_{\mathrm{c}}\right|_{\varphi^{*}=0}=\frac{T^{2}}{(1-\sigma)\left(T-T_{\mathrm{c}}\right)}=\mathcal{O}(1)
$$

while otherwise

$$
\left.\langle I\rangle_{\mathrm{c}}\right|_{\varphi^{*}(\sigma, T)>0}=\frac{N T^{2} \varphi^{* 2}}{2 \eta}=\mathcal{O}(N) .
$$

This last case corresponds to an extensive wave intensity compatible with trapping a macroscopic fraction of the particles. One can thus show that, in the $\omega_{0}(=1)>\sigma$ regime, there exists a second order phase transition for the self-consistent wave-particle system, with the wave kinetic intensity $\psi=I / N$ as order parameter. This phase transition can be viewed as a transition between a trapping regime when $T>T_{\mathrm{c}}$ and an almost-free particles regime when $T<T_{\mathrm{c}}$. We shall now relate O'Neil's dynamical threshold to this phase transition, expressing then a result asymptotic in time.

\section{Revisiting O'Neil's threshold}

Let us consider a finite initial wave intensity $\psi_{0}$ and a distribution function $f_{0}(v)$ - the simplest possible one, i.e. linear — decreasing, of which we are going to vary the slope and thereby the Landau linear damping rate $\gamma_{\mathrm{L}}$. Due to properties of locality in velocity space, the wave only interacts with those particles that are not too far from the separatrices. It can be shown that the particles whose velocities are distant of at least $4 \omega_{B 0}$ from $\omega_{0} / k(=1)$ evolve on KAM tori for the 1.5 degrees of freedom effective Hamiltonian associated to (4), so that they mark the separation between the "active" particles and particles belonging to plasma bulk. Let us therefore put $\Delta=4 \omega_{B 0}=4\left(2 \eta \psi_{0}\right)^{1 / 4}$, the half-width of the resonant domain. We define then the linear $f_{0}(v)$ as (with $a<0)$ :

$$
f_{0}(v)=\left\{\begin{array}{lc}
a v+b & \text { if } v \in[1-\Delta ; 1+\Delta] \\
0 & \text { otherwise }
\end{array}\right.
$$

The normalization of $f_{0}(v)$ gives $b=(2 \Delta)^{-1}-1$, whereas its positivity requires that

$$
-\left(2 \Delta^{2}\right)^{-1}<a
$$

Then one obtains

$$
\begin{aligned}
\langle v\rangle_{0} & =\frac{2}{3} a \Delta^{3}+1 \\
T_{0} & =-\frac{\Delta^{2}}{9}\left(4 a^{2} \Delta^{4}-3\right) .
\end{aligned}
$$

Let us now rewrite the condition $T>T_{\mathrm{c}}$ necessary to obtain the "quasi-free particles", ballistic-like regime. One gets

$$
T>T_{\mathrm{c}} \Leftrightarrow Q(a)>\frac{27}{16} \eta \Delta^{-9}
$$

where $Q$ is the polynomial function of third degree defined by

$$
Q(x)=\left(x-c_{+}\right)\left(x-c_{-}\right)(x-c)
$$

with $c_{ \pm}:=c \pm \sqrt{3} /\left(2 \Delta^{2}\right)$ and $c:=-3 \Delta /\left(4^{5} \eta\right)$. 
It is also necessary that $\sigma<\omega_{0}$ i.e. $\langle v\rangle_{0}+\psi_{0}<1$ so that

$$
a<c=-3 \Delta /\left(4^{5} \eta\right)
$$

Besides, $Q^{\prime}(x)$ vanishes for $d_{ \pm}=c \pm 1 /\left(2 \Delta^{2}\right)$, the relative maximum of $Q$ being reached in $d_{-}$. However, from (12)-(15) and since $d_{-}<-\left(2 \Delta^{2}\right)^{-1}$, one observes a phase transition for this particular class of initial distribution functions, if and only if $Q\left(-\left(2 \Delta^{2}\right)^{-1}\right)>\frac{27}{16} \eta \Delta^{-9}$.

The necessary condition $Q\left(d_{-}\right)>\frac{27}{16} \eta \Delta^{-9}$ is easier to formulate and is equivalent to ${ }^{1}$

$$
\Delta>3 \times 2^{-2 / 3} \eta^{1 / 3}
$$

Here one obtains a condition on the width of the beam. For our picture to be valid, one has to exclude the case of a cold beam : in this singular case, even an infinitesimal initial wave amplitude will not be damped to zero. Interestingly, it turns out that the wave-particle interaction model "à la O'Neil" depicted here definitely differs from the classic study of the Landau damping of a perturbative wave of infinitesimal amplitude. Our framework is non-trivial, because, if the wave is effectively damped to zero asymptotically, its long time behavior (in the kinetic limit) should be governed by Isichenko's analysis [2] due to unavoidable secularities and non-linearities [13]. Thus one can conjecture a very slow, asymptotically algebraic, damping of the wave amplitude towards zero, that is consistent with existing numerical simulations for large $N$.

\section{Conclusion}

Using equilibrium statistical mechanics for the wave-particle model enables to fix in a quantitative way O'Neil's threshold for Landau damping. However, the dynamical chaos available in order to sweep the phase space and to ensure ergodicity is weak in the case of a single Langmuir wave. It seems therefore useful to derive Gibbs thermodynamics for the generic case of a continuum of waves : the existence of strong resonance overlaps should indeed produce in this case a strong, large scale, chaos [15].

Discussions with participants to CEMRACS'99 and members of GdR SParCh are gratefully acknowledged. It is a pleasure to thank F. Coquel and S. Cordier for organizing the workshop at CIRM-Luminy.

\section{REFERENCES}

[1] Landau L 1946, J. Phys. (U.S.S.R.) 1025.

[2] Isichenko M B 1997, Phys. Rev. Lett. 782369.

[3] Lancellotti C and Dorning J J 1998, Phys. Rev. Lett. 80 5236; M B Isichenko 1998, ibid. 5237.

[4] Manfredi G 1997, Phys. Rev. Lett. 792815.

[5] Lancellotti C and Dorning J J 1998, Phys. Rev. Lett. 815137.

[6] Dawson J 1961, Phys. Fluids 4869.

[7] O'Neil T M 1965, Phys. Fluids 82255.

[8] O'Neil T M, Winfrey J H and Malmberg J H 1971, Phys. Fluids 141204.

[9] Mynick H E and Kaufman A N 1978, Phys. Fluids 21653.

[10] Brodin G 1997, Am. J. Phys. 6566.

[11] Antoni M, Elskens Y and Escande D F 1998, Phys. Plasmas 5841.

[12] Firpo M-C and Elskens Y 2000, Phys. Rev. Lett. 843318.

[13] Escande D F, Zekri S and Elskens Y 1996, Phys. Plasmas 33534.

[14] Firpo M-C and Elskens Y 1998, J. Stat. Phys. 93 193; Elskens Y and Firpo M-C 1998, Phys. Scr. T75 169.

[15] Escande D F, Kantz H, Livi R and Ruffo S 1994, J. Stat. Phys. 76605.

\footnotetext{
${ }^{1}$ It is easy to check that this condition is stronger than the condition $-\left(2 \Delta^{2}\right)^{-1}<c$ deduced from (12) and (15).
} 